\title{
Scoliosis in Patients with Parkinson's Disease
}

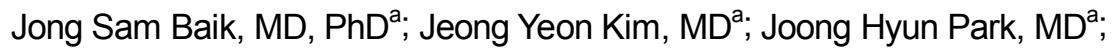 \\ Sang Won Han, MD'; Jae Hyeon Park, MD, PhD'; Myung Sik Lee, MD, PhD \\ Department of ${ }^{a}$ Neurology, Sanggye Paik Hospital, Inje University College of Medicine, Seoul, Korea \\ Department of ${ }^{b}$ Neurology, Kangnam Severance Hospital, Yonsei University College of Medicine, Seoul, Korea
}

\author{
Received January 2,2009 \\ Revised April 30, 2009 \\ Accepted May 4, 2009

\section{Correspondence} \\ Myung Sik Lee, MD, PhD \\ Department of Neurology, \\ Kangnam Severance Hospital, \\ Yonsei University \\ College of Medicine, \\ 612 Eonju-ro, Gangnam-gu, \\ Seoul 135-720, Korea \\ Tel $+82-2-2019-3322$ \\ Fax $+82-2-3462-5904$ \\ E-mail mslee@yuhs.ac
}

Background and Purpose Scoliosis is more common in patients with Parkinson's disease (PD) than in the general elderly population. We compared clinical characteristics between PD patients with and without scoliosis, to identify the relationship between the direction of scoliosis and the laterality of the dominant symptoms of PD. We also studied the associations between dopaminergic pharmacotherapy and scoliosis (defined by a spinal curvature deviation of $10^{\circ}$ or larger).

Methods The study population comprised 97 patients (42 men and 55 women) with idiopathic PD. All of the patients submitted to a whole-spine scanograph to allow measurement of the degree of scoliosis by Cobb's method.

Results True scoliosis was found in 32 of the 97 PD patients, and was observed more frequently in women than in men ( 28 vs. 4 , respectively; $p=0.006$ ). The age of patients without scoliosis was significantly lower than that of those with scoliosis $(66.5 \pm 9.2$ years vs. $72.8 \pm 7.3$ years, respectively, mean $\pm \mathrm{SD}, p<0.001)$. There was no correlation between PD symptom laterality and scoliosis. The rate of occurrence of scoliosis did not differ between de novo and levodopa (Ldopa)-treated patients.

Conclusions We suggest that neither L-dopa treatment nor the laterality of the initial symptoms of PD is related to the appearance of scoliosis.

J Clin Neurol 2009;5:91-94

\section{Introduction}

Scoliosis is defined as lateral curvature of the spine with vertebral rotation leading to asymmetric deformity of the trunk. It occurs more frequently in patients with Parkinson's disease (PD) than in the general population. ${ }^{1-4}$

Several clinical and animal studies have reported that the direction of scoliosis is congruous with the laterality of the major signs and symptoms of $\mathrm{PD},{ }^{3,5-7}$ suggesting that scoliosis is closely associated with nigrostriatal dopamine deficiency. However, contradictory results have also been reported. ${ }^{8}$ The relationship between dopaminergic pharmacotherapy and the direction of scoliosis in PD is also poorly understood.

We divided PD patients into two groups according to the degree of spinal curvature: those with spinal asymmetry (non scoliosis) and those with true scoliosis. We compared the clinical characteristics of the two groups and studied the rela- tionship between the laterality of PD symptoms and the direction of scoliosis. We also studied the association between dopaminergic pharmacotherapy and the risk of developing scoliosis in patients with PD.

\section{Methods}

\section{Patients}

This prospective study was carried out at an outpatient clinic of the Movement Disorders Clinics of Sanggye Paik Hospital (a tertiary, university-affiliated medical center in Seoul), between January 1 and December 31, 2007. This study included PD patients fulfilling United Kingdom Brain Bank Criteria, ${ }^{9}$ and excluded those with atypical parkinsonism, secondary parkinsonism, long-standing idiopathic scoliosis, and previous cerebral infarction or head trauma. The degree of scoliosis was assessed using whole-spine radiography, and 
the Unified Parkinson's Disease Rating Scale Motor Score (UPDRS III $)^{10}$ and the Hoehn and Yahr (H\&Y $)^{11}$ scores were evaluated. The clinical characteristics of the cohort were determined (including age at initial evaluation, gender, initial symptom side, age of PD symptom onset, surgery and back pain history, and drug treatment) by questioning the subjects and their caregivers, and studying their medical records. Patients who were medicated with levodopa (L-dopa) during ON time were identified. Informed, written consent to participate was obtained from the patients after they had been given a complete description of the whole-spine scanograph procedure.

\section{Radiological examination and analysis}

Scoliosis was evaluated from an upright posteroanterior radiograph of the entire spine of each patient. If a patient was unable to stand upright for a whole-spine scanograph, a simple spine X-ray was performed. The degree, location, and direction of the scoliosis curve were assessed. Digital X-ray films and the Cobb method were used to measure the curve, ${ }^{12}$ and the degree of curvature was measured automatically with the aid of a digital X-ray viewing system (Fig. 1). Using the findings, we divided patients into those with normal or spinal asymmetry (deviation of less than $10^{\circ}$ ), and those with scoliosis (deviation of $10^{\circ}$ or more) ${ }^{13}$ All digital films were



Fig. 1. A 65-year-old female de novo patient, with a 1-year history of bradykinesia on the left side exhibited right-sided scoliosis in the lumbar area with a deviation $16.32^{\circ}$ (as assessed using the Cobb method and whole-spine scanography). evaluated by a radiologist. Curve location was defined by its center, known as the apex, which is the most lateral disc or vertebra of the curve. The scoliosis cases were then subdivided according to the apical vertebra into cervical, thoracic, and lumbar cases. The direction of curvature was defined as direction of convexity.

Data were analyzed with the statistical package SPSS for Windows (version 12.0, SPSS, Chicago, Illinois, USA). Demographic data comparisons between the spinal asymmetry and scoliosis groups were conducted using unpaired $t$-tests and $\chi^{2}$ statistics. The level of statistical significance was set at $p<0.05$.

\section{Results}

A total of 97 PD patients (42 men and 55 women) comprised the cohort for this study. The age of onset of PD was $65.0 \pm$ 9.7 years (mean $\pm \mathrm{SD}$; range $37-88$ years), and the duration of the disease was $39.9 \pm 30.0$ months (range 1-103 months). The H\&Y stage was $1.6 \pm 0.7$ (range 1-3), and the UPDRS III score was $22.5 \pm 14.7$ (range 4-83). Thirty-two of the 97 patients $(33 \%)$ had scoliosis: $2(5.6 \%)$ at the cervico thoracic junction, $7(19.4 \%)$ in the thoracic spine, $13(36.1 \%)$ at the thoraco lumbar junction, and 14 (38.9\%) in the lumbar spine. Four of the 32 patients had double curves. The side on which PD symptoms were more severe was right in 50 patients and left in 47. In 38 of 97 patients the initial dominant symptom was akinetic-rigid syndrome, in 30 it was tremor, and in 29 it was a combination of both.

The various clinical characteristics were compared between patients with and without scoliosis. Patients with scoliosis were significantly older ( $72.8 \pm 7.3$ years) than those without (66.5 \pm 9.2 years, $p<0.001$ ), and scoliosis was observed in women 7 times more frequently than in men ( 28 women vs. 4 men, $p=0.006$ ). There were no significant differences in any of the other clinical characteristics (Table 1). The relationship between dopaminergic pharmacotherapy and scoliosis was evaluated by comparing de novo and L-dopa-treated patients; with the exception of disease duration there were no significant differences between the clinical features of the two groups (Table 2). The direction of scoliosis was concordant with PD laterality in 8 out of 18 treated patients (44.4\%) and in 8 out of 14 de novo patients $(57.1 \%)$.

\section{Discussion}

Scoliosis is a structural lateral curvature of the spine with a rotator component. The incidence of scoliosis among healthy elderly patients varies from $6 \%$ to $30 \%,{ }^{1,2}$ but this figure rise in PD ranging from $43 \%$ to $90 \%{ }^{3,4,8}$ Ashour and Jankovic ${ }^{14}$ 
Baik JS et al.

Table 1. Clinical characteristics of non-scoliosis and true scoliosis patients

\begin{tabular}{|c|c|c|c|}
\hline & Non-scoliosis & True scoliosis & $\mathrm{p}$ \\
\hline Total number of patients & 65 & 32 & \\
\hline Gender (males: females) & $38: 27(1: 0.71)$ & $4: 28(1: 7.00)$ & $<0.001$ \\
\hline Age, years (mean $\pm S D$ and range) & $66.53 \pm 9.15(43-85)$ & $72.75 \pm 7.29(55-90)$ & $<0.001$ \\
\hline Duration of PD, month (mean $\pm S D$ and range) & $40.65 \pm 29.72(4-98)$ & $36.91 \pm 29.07(1-95)$ & 0.559 \\
\hline \multicolumn{4}{|l|}{ Initial PD symptom, n (\%) } \\
\hline Tremor & $18(27.7 \%)$ & $12(37.5 \%)$ & 0.454 \\
\hline Akinetic-rigid & $26(40.0 \%)$ & $12(37.5 \%)$ & 0.987 \\
\hline Tremor+akinetic-rigid & $21(32.3 \%)$ & $8(25.0 \%)$ & 0.615 \\
\hline H\&Y stage (mean \pm SD and range) & $1.52 \pm 0.61(1-3)$ & $1.64 \pm 0.73(1-3)$ & 0.379 \\
\hline UPDRS III (mean \pm SD and range) & $22.67 \pm 12.21(5-51)$ & $21.89 \pm 20.38(4-83)$ & 0.847 \\
\hline History of back pain, $n(\%)$ & $22 / 42(52.4 \%)$ & $15 / 20(75.0 \%)$ & 0.156 \\
\hline Previous surgery, $n(\%)$ & $6 / 54(11.2 \%)$ & $4 / 28(14.3 \%)$ & 0.959 \\
\hline De novo patients, $n(\%)$ & $29 / 65$ (44.6\%) & $14 / 32$ (43.8\%) & 0.887 \\
\hline L-dopa-treated patients, n (\%) & 35 (53.9\%) & $18(56.3 \%)$ & 0.995 \\
\hline
\end{tabular}

PD: Parkinson's disease, H\&Y: Hoehn and Yahr, UPDRS III: Unified Parkinson's Disease Rating Scale Motor Score, L-dopa: levodopa.

Table 2. Clinical characteristics of de novo and L-dopa-treated patients

\begin{tabular}{|c|c|c|c|}
\hline & De novo & L-dopa-treated & $p$ \\
\hline Total number of patients & 43 & 54 & \\
\hline Gender (males: females) & $18: 25(1: 1.39)$ & $24: 30(1: 1.25)$ & 0.93 \\
\hline Age, years (mean $\pm S D$ and range) & $67.12 \pm 9.91(43-90)$ & $69.76 \pm 8.17(42-85)$ & 0.153 \\
\hline Duration of PD, months (mean \pm SD and range) & $19.84 \pm 18.81(1-83)$ & $55.00 \pm 26.98(6-98)$ & $<0.001$ \\
\hline \multicolumn{4}{|l|}{ Initial PD symptom, n (\%) } \\
\hline Tremor & $12(27.9 \%)$ & $18(33.3 \%)$ & 0.726 \\
\hline Akinetic-rigid & $14(32.6 \%)$ & $24(44.4 \%)$ & 0.33 \\
\hline Tremor+akinetic-rigid & $17(39.5 \%)$ & $12(22.3 \%)$ & 0.106 \\
\hline H\&Y stage (mean \pm SD and range) & $1.44 \pm 0.64(1-3)$ & $1.64 \pm 0.64(1-3)$ & 0.14 \\
\hline UPDRS III (mean \pm SD and range) & $21.65 \pm 17.46(5-83)$ & $23.10 \pm 12.32(4-51)$ & 0.683 \\
\hline History of back pain, $n(\%)$ & $12 / 19(63.2 \%)$ & $25 / 43(58.1 \%)$ & 0.923 \\
\hline Previous surgery, $n(\%)$ & $5 / 28(17.9 \%)$ & $5 / 52(9.6 \%)$ & 0.474 \\
\hline Scoliosis patients, n (\%) & $14 / 43(32.6 \%)$ & $18 / 54(33.3 \%)$ & 0.885 \\
\hline
\end{tabular}

recently reported 14 patients with scoliosis among a total of 164 PD patients (i.e., $8.5 \%$ ). Their study was retrospective and radiological evaluation was not performed. However, the present study cohort of 97 included 32 PD patients (33\%) with scoliosis with a deviation of $10^{\circ}$ or more.

Genetic, hormonal, biomechanical, and neuromuscular factors have been proposed as possible causes of scoliosis in PD, but its exact pathophysiology is not yet understood. ${ }^{15}$ In accordance with the frequent dystonia reported in women with $\mathrm{PD},{ }^{16}$ the present study showed that scoliosis occurred seven times more frequently in women with PD than in their male counterparts. Our data also show that old-age onset of PD is another risk factor for scoliosis in PD.

Duvoisin and Marsden showed that 19 of 21 consecutive unoperated PD patients had the same direction of postural deviation, which was correlated significantly with the latera- lity of the major signs and symptoms of their parkinsonism. ${ }^{3}$ They considered the scoliosis to be analogous to that observed in animals after unilateral destruction of the dopaminergic nigrostriatal pathway arising from the zona compacta of the substantia nigra. ${ }^{17}$ Recent studies have shown that experimental central nervous system lesions induce scoliosis. ${ }^{6,7}$ Axial dystonias, such as camptocormia, Pisa syndrome, or scoliosis, could be caused by bilateral involvement of the brain stem ascending dopamine pathways. ${ }^{18}$ However, the present study found that the laterality of PD symptoms and the severity of parkinsonian deficits were not related to the direction and development of scoliosis. Similar to the present study, other study showed no relationship between the direction of scoliosis and laterality of Parkinsonian signs. ${ }^{8}$ Although it has been shown previously that the frequency and severity of dystonic posture are correlated with the duration of L-dopa 
treatment, ${ }^{16}$ we could not find any relationship between scoliosis and medical treatment. These findings suggest that nigrostriatal dopamine deficiency is not a major causative factor in the development of scoliosis.

Degenerative scoliosis is usually associated with a variable degree of loss of lordosis, axial rotation, lateral listhesis, and spondylolisthesis. ${ }^{13}$ Other than scoliosis, our patients had no spinal abnormalities. The other possible explanation is osteoporosis; however, some of the patients included in this study, especially those with scoliosis in the lower lumbar area, may have had degenerative scoliosis. Osteoporosis and lumbar scoliosis are known to be diseases of the elderly that occur particularly frequently in women and often occur together. ${ }^{19,20}$ Although some studies have shown no correlation between osteoporosis and scoliosis, ${ }^{1,21}$ others have shown that the prevalence of scoliosis is higher among the elderly with osteoporosis. ${ }^{19,20,22}$ However, the present study showed that only $38.9 \%$ of patients (14 of 36 lesions) had scoliosis in the lumbar area, which is the site most frequently affected by the degenerative scoliosis associated with osteoporosis.

Our study has some limitations. One is that most of our patients had relatively mild PD symptoms (mean H\&Y stage 1.6). This makes it difficult to compare our findings with those of patients with advanced PD. Another limitation of our study is that we did not include control population. Also, it's difficult to discern the base rate of scoliosis by age, because our data may contain many elderly individuals with mild scoliosis.

In spite of these limitations, our results suggest that scoliosis in PD is not related to any of the clinical manifestations of PD, including disease severity (H\&Y stage, UPDRS III scores), and that the direction of scoliosis convexity is not related to the laterality of initial PD symptoms. Although the incidence and severity of dystonic posture are correlated with L-dopa treatment in PD-associated dystonia, we could not find any correlation with L-dopa treatment in PD-associated scoliosis.

In conclusion, scoliosis in patients with PD is common, most having a single curvature. Scoliosis is more common in women than men, and the onset age of PD in patients with scoliosis is older than in those without. The presence of scoliosis in PD patients is not related to the mean duration of PD, initial dominant symptoms, UPDRS III scores, and L-dopa treatment. Although we did not compare all of the clinical features between the concordance and non-concordance groups, it appears that the direction of scoliosis may not be correlated with the laterality of the major signs and symptoms of parkinsonism or with L-dopa treatment.

\section{Acknowledgments}

This study has been received financial support from Inje University Research Foundation.

\section{REFERENCES}

1. Robin GC, Span Y, Steinberg R, Makin M, Menczel J. Scoliosis in the elderly: a follow-up study. Spine 1982;7:355-359.

2. Vanderpool DW, James JI, Wynne-Davies R. Scoliosis in the elderly. J Bone Joint Surg AM 1969;51:446-455.

3. Duvoisin RC, Marsden CD. Note on the scoliosis of Parkinsonism. $J$ Neurol Neurosurg Psychiatry 1975;38:787-793.

4. Indo T, Ando K. [Studies on the scoliosis of Parkinsonism (author's transl)]. Rinsho Shin-keigaku 1980;20:40-46.

5. Martin JP. Curvature of the spine in post-encephalitic parkinsonism. J Neurol Neurosurg Psychiatry 1965;28:395-400.

6. Herrera-Marschitz M, Utsumi H, Ungerstedt U. Scoliosis in rats with experimentally-induced hemiparkinsonism: dependence upon striatal dopamine denervation. J Neurol Neurosurg Psychiatry 1990;53:39-43.

7. Lundblad M, Picconi B, Lindgren H, Cenci MA. A model of L-DOPA-induced dyskinesia in 6-hydroxydopamine lesioned mice: relation to motor and cellular parameters of nigrostriatal function. Neurobiol Dis 2004;16:110-123.

8. Grimes JD, Hassan MN, Trent G, Halle D, Armstrong GW. Clinical and radiographic features of scoliosis in Parkinson's disease. $\mathrm{Adv} \mathrm{Neu}$ rol 1987;45:353-355.

9. Hughes AJ, Ben-Shlomo Y, Daniel SE, Lees AJ. What features improve the accuracy of clinical diagnosis in Parkinson's disease: a clinicopathologic study. Neurology 1992;42:1142-1146.

10. Fahn S, Elton RL, Members of the UPDRS Development Committee. Unified Parkinson's disease rating scale. In: Fahn S, Marsden CD, Calne DB, Golstein M, editors. Recent developments in Parkinson's disease, Vol. 2. Florham Park (NJ): Macmillan Health Care, 1987;153164.

11. Hoehn MM, Yahr MD. Parkinsonism: onset, progression and mortality. Neurology 1967;17:427-442.

12. Cobb JR. Outline for the study of scoliosis. Am Acad Orthop Surg 1948;5:261-275.

13. Van Goethem J, Van Campenhout A, van den Hauwe L, Parizel PM. Scoliosis. Neuroimag Clin N Am 2007;17:105-115.

14. Ashour R, Jankovic J. Joint and skeletal deformities in Parkinson's disease, multiple system atrophy, and progressive supranuclear palsy. Mov Disord 2006;21:1856-1863.

15. Lowe TG, Edgar M, Margulies JY, Miller NH, Raso VJ, Reinker KA, et al. Etiology of idiopathic scoliosis: current trends in research. $J$ Bone Joint Surg Am 2000;82-A:1157-1168.

16. Jankovic J, Tintner R. Dystonia and parkinsonism. Parkinsonism Relat Disord 2001;8:109-121.

17. Ungerstedt U. Postsynaptic supersensitivity after 6-hydroxy-dopamine induced degeneration of the nigro-striatal dopamine system. Acta Phsiol Scand Suppl 1971;367:69-93.

18. Péchadre JC, Larochelle L, Poirier LJ. Parkinsonian akinesia, rigidity and tremor in the monkey. Histopathological and neuropharmacological study. J Neurol Sci 1976;28:147-157.

19. Daffner SD, Vaccaro AR. Adult degenerative lumbar scoliosis. Am J Orthop 2003;32:77-82; discussion 82 .

20. Healey JH, Lane JM. Structural scoliosis in osteoporotic women. Clin Orthop Relat Res 1985:216-223.

21. Robin GC. Scoliosis in the elderly: idiopathic or osteoporotic? Clin Orthop Relat Res 1986:311-312.

22. Thevenon A, Pollez B, Cantegrit F, Tison-Muchery F, Marchandise X, Duquesnoy B. Relationship between kyphosis, scoliosis, and osteoporosis in the elderly population. Spine 1987;12:744-745. 